\title{
CONCEPCIONES SOBRE DINERO Y DECISIONES SOBRE SU USO EN TWEENS
}

\section{CONCEPTS OF MONEY AND DECISIONS ABOUT ITS USE IN TWEENS}

\author{
Marianela Denegri C. ${ }^{*}$, Javier Álvarez G.,Jocelyne Sepúlveda A. \\ UNIVERSIDAD DE LA FRONTERA
}

(RECIBIDO: 8-4-2013 ACEPTADO: 20-7-2013)

\begin{abstract}
RESUMEN
El objetivo de esta investigación fue determinar la posible relación existente entre hábitos de consumo (uso del dinero, decisiones que se toman para usarlo e ideas sobre este), nivel socioeconómico y género en tweens. La muestra fue constituida por 468 participantes de la ciudad de Temuco, Chile, cuyas edades fluctúan entre los 10 y 14 años. Se aplicó el Cuestionario de Hábitos de Consumo (Denegri, Lara, Córdova y Del Valle, 2008). Los resultados indican relaciones estadísticamente significativas entre nivel socioeconómico y uso dado al dinero, forma de decidir y concepciones sobre el dinero. La información complementa lo observado en estudios previos, sin embargo se sugiere un estudio longitudinal para observar la evolución de estos hábitos a lo largo del tiempo.
\end{abstract}

Palabras clave: uso del dinero, ideas sobre dinero, tweens, hábitos de consumo.

\begin{abstract}
The purpose of this research was to determine the possible relationship among consumption habits (use of money, decisions about its use and ideas about money), socioeconomic status and gender on tweens. The sample was constituted by 468 participants from city of Temuco, Chile, their ages range between 10 and 14. We applied the Consumer Habits Questionnaire (Denegri, Lara, Cordova, and Del Valle, 2008). The results indicate statistically significant relationships between NSE and use of money, decision of use and ideas about money. The information complements the observed in previous studies. A longitudinal study is suggested to observe the evolution of these habits over time.
\end{abstract}

Keywords: use of money, ideas about money, tweens, consumption habits. 


\section{INTRODUCCIÓN}

En la sociedad actual, caracterizada por el consumismo y en donde el dinero ha adquirido gran importancia no sólo a un nivel estrictamente económico, sino también psicológico, la socialización económica se vuelve un factor de relevancia que puede ayudar a individuos y familias a interactuar con un entorno complejo que presiona hacia el consumo. En este contexto, se vuelve necesario que el individuo, por un lado, sea capaz de manejar informaciones y conocimientos económicos específicos, mientras que por otro lado, pueda desarrollar habilidades, valores y actitudes para inferir en el curso de los eventos económicos cotidianos, anticipándose a tomar decisiones económicas que resulten favorables para sí mismo y su entorno (Denegri, Lara, Córdova y Del Valle, 2008).

Sin embargo, pese a ser un fenómeno tan importante, cuyo ámbito de influencia abarca desde pautas conductuales, actitudes, creencias, informaciones y que impacta fuertemente en la calidad de vida de los individuos, la literatura latinoamericana sobre cómo se expresa la socialización económica en las diferentes etapas de la vida, específicamente durante la infancia y la adolescencia (Denegri et al., 2008), no es aún lo suficientemente amplia. A nivel mundial, a partir de los años 60 comienza la investigación acerca de los patrones de consumo en la infancia y los niños son considerados como un segmento diferenciado del resto de los consumidores, lo que origina crecientes estrategias de mercadotecnia dirigidas a este segmento (McNeal, 1965; McNeal y Yeh, 1990; 1997). La mayoría de los estudios indican la creciente influencia que los niños ejercen en la toma de decisiones de consumo familiar y que cada vez más temprano comienzan a manejar autónoma y cotidianamente dinero para sus gastos (Furnhan y Argyle, 1998; Guber y Berry, 1993; Moschis, 1987; Rose, 1999). Estos fenómenos no sólo se han observado en Occidente, sino también en otros contextos culturales, por ejemplo en Turquía (Ozgen, 2003) donde se observa un rápido incremento de las actividades de consumo en los niños y como éstos toman decisiones independientes para usar su dinero.

Por otro lado, estudios en Chile llevados a cabo el año 2003 por el Consejo Nacional de Televisión y la agencia de publicidad McCann-Erickson, reportaron que durante el año del estudio los niños de entre 8 y 13 años, pertenecientes a la capital del país, recibieron aproximadamente y en su conjunto un total de 749 millones de pesos para sus gastos. Este dinero recibido suele ser usado principalmente para comprar dulces y golosinas en general, además de destinar una parte al ahorro. Resultados similares describen Denegri et al. (2008) respecto a los diversos usos que los niños dan al dinero y cómo varían de acuerdo al nivel socioeconómico (NSE), reportando diferencias significativas entre los niveles observados. De acuerdo al estudio, el uso que los niños generalmente dan a su dinero está destinado a gastos personales, ahorro, diversión, gastos familiares y alimentación. Sin embargo, se observaron diferencias en cada uno de los niveles socioeconómicos observados, por ejemplo, los niños pertenecientes al NSE bajo destinan, principalmente, el dinero para la colación del colegio y ayuda económica en su hogar, gastos usualmente atribuidos a los padres. Por otro lado, niños de NSE alto utilizan su dinero principalmente para la diversión. Los autores explican 
esto debido a las diferencias en la disponibilidad de recursos otorgados por los padres, sumado a prácticas de ahorro más regulares en niños de NSE alto, lo que les permiten mayores oportunidades de consumo.

Es en el estudio de CNTV y McCann-Erickson donde además se comienza a utilizar el concepto de tweens, que surge inicialmente como un concepto asociado al marketing pero que rápidamente pasa a formar parte también de las características que este segmento etáreo hace de la psicología económica y del consumo (CNTV y McCann-Erickson, 2003; Denegri et al., 2008).

El segmento tweens corresponde a aquellos niños de entre 8 y 13 años de edad, quienes presentan una temprana incorporación al mundo del dinero, siendo capaces de decidir, discriminar e influir en la compra o elección de distintos productos para ellos o su familia. Sus padres no suelen ver este tipo de conductas de manera positiva, sin embargo, se asume como parte de la educación que hoy en día deben recibir los niños en esta edad. Además, los tweens se caracterizan por tener un elevado poder adquisitivo, constituyéndose como potentes consumidores, ya sea usando su propio dinero o persuadiendo a sus padres a comprar (CNTV y McCann-Erickson, 2003; Denegri et al., 2008).

El comportamiento de los tweens, como consumidores, tiende a ser más racional y menos impulsivo que lo esperado a su edad, expresado principalmente en la incorporación del concepto de ahorro a su economía personal. Están conscientes de su poder de compra e influencia, además confían en sus capacidades de ahorro y persuasión para conseguir lo que quieren. El principal espacio donde los tweens pueden expresarse como consumidores es el colegio. En este contexto los niños pasaron de llevar la colación desde su hogar a comprarla directamente en sus establecimientos, donde consumen principalmente golosinas y snacks, particularmente en el horario de recreo (CNTV y McCann-Ericsson, 2003; Denegri et al., 2008).

Paralelo a su rol como consumidores, los tweens se presentan como un grupo capaz de esparcir las modas y las cosas consideradas atractivas, relacionado con su interés por probar productos nuevos, compararlos, innovar y estar actualizados. Les interesa informarse, opinar e interactuar, además de ser escuchados y tomados en serio, construyendo una concepción propia del mundo basada tanto en lo que perciben en sus familias, como en lo que observan en los medios de comunicación, principalmente a través de la televisión e internet. Para estos niños, llamados por otros autores "nativos digitales" (Prensky, 2001), la tecnología juega un rol importante en la forma que se reúnen, comparten y construyen su identidad cerca de sus pares, la consideran una herramienta natural en su diario vivir y se relacionan con ella de manera instrumental e interactiva, ya que por un lado les permite realizar sus tareas, entretenerse e informarse, mientras que por otro, pueden interactuar con sus pares, incluso con programas de televisión (CNTV y McCann-Ericsson, 2003; Prensky, 2001).

Esta generación de nuevos consumidores posee además otra particularidad que la diferencia de otros segmentos, puesto que agrupa en sí misma tres grupos de mercado, lo que además les hace ser un grupo muy apetecido por las estrategias 
de mercadotecnia. Por una parte, al volverse fuertes demandantes de diversos productos y servicios, constituyen un Mercado Primario de consumidores, facilitado por su tiempo libre, su curiosidad, su permeabilidad frente a la información y lo más llamativo, y las grandes sumas de dinero que manejan actualmente, las cuales van aumentando. Por otro lado, poseen las característica de ser un Mercado de Influencia dado que, mediante la persuasión, son capaces de orientar el gasto de sus padres en beneficio propio, influyendo positivamente en las decisiones económicas que los padres toman, por ejemplo a qué lugares vacacionar, qué productos comprar, dónde comprar, etc. Por último, los tweens representan un Mercado Futuro, ya que al alcanzar una edad de mercado para determinada empresa, proporcionarán un flujo constante de nuevos consumidores de aquellas marcas a las que se han habituados desde pequeños, constituyendo un proceso conocido como fidelización (McNeal, 2000; Denegri et al., 2008).

La edad en la que se sitúan los tweens, es de mucha importancia, dado que entre los 9 y 11 años se establecen muchas de las actitudes y hábitos de consumo que continuarán presentes en su vida como adultos (Ozgen, 2003). Por ello, es importante estudiar sus hábitos de consumo, ya que este conocimiento aporta al desarrollo de iniciativas efectivas que faciliten su cada vez más temprana socialización económica, orientada al desarrollo de actitudes y conductas económicas "saludables", tales como estrategias de ahorro, análisis crítico de los medios, actividades de pre y post-compra, etc. (McNeal, 2000; Denegri y Martínez, 2004), con el fin de formar ciudadanos informados más que consumidores pasivos.

En función de los antecedentes planteados, la pregunta de investigación que orientó esta investigación fue: ¿Cuál es la relación existente entre los hábitos de consumo que presentan los tweens, su nivel socioeconómico y género? Para responder a esto, se planteó como objetivo general determinar la posible relación existente entre los hábitos de consumo (uso del dinero, decisiones que se toman para usarlo y concepciones sobre este), nivel socioeconómico y género. Los objetivos específicos fueron (a) establecer la relación entre el NSE y uso de dinero, (b) establecer la relación entre el NSE y decisiones sobre el uso, (c) establecer la relación entre el NSE y concepciones sobre el dinero, (d) establecer la relación entre el género y uso de dinero, (e) establecer la relación entre el género y decisiones sobre el uso, y (f) establecer la relación entre el género y concepciones sobre el dinero.

\section{MÉTODO}

\section{Participantes}

La muestra fue constituida por 468 niños de entre 10 y 14 años $(M=11,18$; DS $=$ $0,585)$, de los cuales 228 fueron hombres y 240 mujeres, estudiantes de $6^{\circ}$ año de Educación General Básica de diversos establecimientos educacionales de la ciudad de Temuco, Chile. Según la distribución por NSE, el 23,3\% de participantes pertenece al NSE bajo, el 31,6\% al NSE medio-bajo, el 27,1\% al NSE medio-alto y el $17,9 \%$ correspondieron al NSE alto. 


\section{Procedimiento de muestreo}

La población objetivo consideró a todos los alumnos que cursaron sexto año de Educación General Básica, en ciudades multifinancieras con más de 200.000 habitantes, siendo la ciudad de Temuco la única dentro de la región que se ajusta a los criterios. El procedimiento de muestreo fue bietápico. En la primera fase, de carácter no probabilístico e intencionado, se seleccionaron establecimientos educacionales que cumplían con determinados criterios de inclusión, tales como matrícula mixta, localización urbana, sobre $99 \%$ de matrículas con procedencia urbana, un mínimo de dos secciones por nivel (p. e.: $6^{\circ}$ A y B) y asignación a secciones según criterios históricos y no por rendimiento. A continuación, el conjunto de establecimientos que correspondían a los criterios se dividió en tres grupos según dependencia: Municipal, Particular Subvencionado y Particular Pagado. Finalmente, se obtuvieron un grupo de establecimientos homogéneos en los criterios de inclusión. La segunda etapa consistió en la selección aleatoria de dos establecimientos por cada tipo de dependencia, obteniéndose 8 establecimientos educacionales en total. Este procedimiento fortalece el proceso de muestreo e impacta positivamente en la validez externa del estudio. Posteriormente, se llevó a cabo el procedimiento para conseguir la muestra de estudiantes, la cual se llevó a cabo mediante la selección aleatoria de cursos en cada colegio.

\section{Diseño}

Esta investigación fue desarrollada bajo un enfoque cuantitativo, no experimental. El diseño fue transversal, dado que los datos fueron tomados en un momento determinado, y de acuerdo a los objetivos planteados fue de tipo descriptivocorrelacional (Hernández, Fernández, y Baptista, 1994).

\section{Instrumento}

El instrumento utilizado fue el Cuestionario de Hábitos de Consumo desarrollado por Denegri et al. (2008). Este instrumento consta de 10 ítems de selección múltiple y 6 de respuesta abierta, que no serán incluidos en este estudio pues no forman parte de los objetivos propuestos para la investigación, y está orientado a indagar preferencias y prácticas de consumo, además de concepciones sobre el dinero y el uso dado a éste.

\section{Procedimiento de análisis}

En primer lugar se emplearon estadísticos descriptivos y análisis de frecuencias para caracterizar las principales conductas de consumo. En segundo lugar, se utilizó la prueba Chi cuadrado $\left(\mathrm{X}^{2}\right)$ para medir la relación entre las distintas variables. Con el fin de medir la fuerza de las relaciones se recurrió el coeficiente de contingencia (C). Se consideraron valores de significación (p) menores a 0,05 como criterio para identificar las relaciones significativas. Todos estos análisis fueron llevados a cabo mediante un programa de análisis estadístico. 
CONCEPCIONES SOBRE DINERO Y DECISIONES SOBRE SU USO EN TWEENS

\section{RESULTADOS}

\section{Niños que reciben dinero y cantidad de dinero recibida}

El $92,1 \%$ de los niños dijeron recibir dinero de sus padres, reportando recibir cada vez en promedio 2633,29 pesos (U\$ 5) $(\mathrm{DS}=4140,37)$, siendo la menor cantidad recibida equivalente a 100 pesos, mientras que la mayor a 50000 pesos (U\$10). De acuerdo al NSE, los niños de NSE bajo reciben $\$ 2343,88$; los de estratos medio-bajo y medio-alto reciben $\$ 2779,63$ y $\$ 2308,42$ respectivamente, mientras que los niños de NSE alto reciben $\$ 2308,42$.

\section{Frecuencia con la que los padres dan dinero a sus hijos y NSE}

El 23,3\% de los niños de los estratos medios reportaron recibir dinero todos los días, de manera significativa respecto a los demás NSE $\left(\mathrm{x}^{2}=8,331 ; \mathrm{p}=0,040\right.$; $\mathrm{c}=0,132 ; \mathrm{p}=0,040)$. Por otro lado, el $22 \%$ de los niños de NSE bajo indicó recibir dinero de manera semanal, seguido por el 13,5\% de los niños de NSE mediobajo y el 11,5\% de los niños de NSE alto $\left(x^{2}=12,310 ; p=0,006 ; c=0,162 ; p=\right.$ 0,006). Por último, el $31,1 \%$ de los niños de NSE medio-bajo indicaron recibir dinero mensualmente $\left(x^{2}=13,165 ; p=0,004 ; c=0,165 ; p=0,004\right)$ (Tabla 1).

\section{Frecuencia con la que los padres dan dinero a sus hijos y género}

$\mathrm{Al}$ establecer la relación por género, se observaron resultados significativos entre esta variable y la entrega mensual de dinero $\left(\mathrm{x}^{2}=6,962 ; \mathrm{C}=0,121 ; \mathrm{p}=0,008^{*}\right)$, donde el $27,2 \%$ de los hombres marcó esta opción, frente al 17,1\% de las mujeres. Por otro lado, una relación significativa se observó entre género y la entrega de dinero sólo cuando el niño lo pide $\left(x^{2}=19,054 ; C=0,194 ; p=0,000^{*}\right)$, donde el 58,3\% de las mujeres marcó esta alternativa (Tabla 2).

\section{Relación entre el uso dado al dinero y NSE}

Se observó una relación significativa entre el NSE y la práctica de ahorro $\left(x^{2}=22,828 ; c=0,216 ; p=0,000\right)$, siendo reportada por el $66,9 \%$ de los niños de NSE medio-alto y el 65,5\% de NSE alto. El 23,9\% de los niños de NSE bajo indicó usar su dinero para comprar cosas del colegio $\left(x^{2}=42,658 ; c=0,289 ; p=0,000\right)$. Por otro lado, el 6,4\% de los niños de NSE bajo reportó también comprar cosas para la casa, a pesar de ser menor el porcentaje, esta relación fue significativa $\left(\mathrm{x}^{2}=11,314 ; \mathrm{c}=0,154 ; \mathrm{p}=0,010\right)($ Tabla 3$)$.

Se observó una relación significativa $\left(x^{2}=27,229 ; p=0,027 ; c=0,241 ; p=0,027\right)$ al indagar la relación entre el NSE y el consumo de cosas que no están incluidas en las destinadas al colegio y al hogar. Los niños de NSE bajo consumen principalmente en juguetes y golosinas, mientras que aquellos niños de NSE medios gastan su dinero en vestimentas y regalos. Por otro lado, los niños de NSE alto destinan su dinero a libros, revistas y golosinas (Tabla 4). 


\section{Relación entre el uso dado al dinero y género}

No se observaron relaciones significativas entre el género y esta dimensión. Sin embargo, se observó una relación significativa al indagar cuáles son los productos que compran hombres y mujeres con el dinero que le dan sus padres $\left(\mathrm{x}^{2}=26,732\right.$; $\mathrm{C}=0,232 ; \mathrm{p}=0,000)$. Los hombres suelen comprar juegos y juguetes, mientras que las niñas compran vestimentas, libros y regalos (Tabla 5 y 6 ).

\section{Relación entre las decisiones de uso y NSE}

El análisis realizado arrojó una relación significativa al contrastar esta variable con el NSE de los participantes $\left(x^{2}=32,177 ; p=0,000\right)$, mientras que el grado de la relación, obtenido a través del coeficiente de contingencia, fue baja $(c=0,254$; $\mathrm{p}=0,000$ ). Se observan diferencias en la forma de decidir qué hacer con el dinero, el $17,8 \%$ de los niños de NSE bajo deciden en conjunto con sus padres, estos últimos deciden, en algunos casos, qué harán los niños con el dinero obtenido. Por otro lado, los niños de NSE medio-alto y alto $(15,2 \%$ y $16,7 \%$ respectivamente) conversan con sus padres (Tabla 7).

\section{Relación entre las decisiones de uso y género}

No se observaron relaciones significativas entre género y la forma en que los niños deciden usar su dinero.

\section{Relación entre las concepciones sobre el dinero y el NSE}

Un $64 \%$ de los niños del NSE bajo reportaron que el dinero sirve para comprar, observándose una relación estadísticamente significativa entre esta noción y el NSE $\left(x^{2}=21,251 ; p=0,000 ; c=0,213 ; p=0,000\right)$. El 43,2\% de los niños de NSE medio-bajo y el $33,1 \%$ de los niños provenientes del NSE medio-alto indicaron que el dinero es importante, diferenciándose de manera significativa respecto a los demás NSE $\left(\mathrm{x}^{2}=25,290 ; \mathrm{p}=0,000 ; \mathrm{c}=0,232 ; \mathrm{p}=0,000\right)$. Por otro lado, un $21,4 \%$ de los niños de NSE alto indican que el dinero no es importante $\left(x^{2}=7,897\right.$; $\mathrm{p}=0,048 ; \mathrm{c}=0,130 ; \mathrm{p}=0,048)($ Tabla 8).

\section{Relación entre las concepciones sobre el dinero y el género}

No se observaron relaciones significativas entre el género y las opiniones que los niños tienen sobre el dinero.

\section{DISCUSIÓN}

Los resultados complementan lo encontrado por otros autores sobre el comportamiento de los tweens en el ámbito económico, y las diferentes formas en que lo expresan como consumidores insertos en la sociedad actual. En consistencia con estos estudios, se confirma la presencia del ahorro como una práctica presente 
en los NSE medio-alto y alto, y algunas prácticas de uso del dinero observadas anteriormente (Denegri, et al., 2008).

De acuerdo a las relaciones observadas entre el nivel socioeconómico y los hábitos de consumo estudiados, es posible describir perfiles de pautas de consumo claramente diferenciados entre los niños. En primer lugar, se puede caracterizar a los tweens de NSE bajo como niños que reciben dinero de sus padres de manera semanal y con menor autonomía en las decisiones sobre su uso, siendo el estrato donde los padres presentan el mayor porcentaje de influencia en la decisión sobre el uso que los niños deben dar a su dinero, ya sea conversando o decidiendo directamente por ellos. Otro aspecto diferenciador se observa en el destino que los niños dan al dinero, ya sea recibido de sus padres o generado directamente a través de trabajo infantil. En este estrato, el dinero recibido por los niños es destinado principalmente para comprar cosas necesarias para la escuela y la casa, conducta reportada por Denegri et al. (2008), quienes describen el rol de proveedores que, particularmente, los varones ejercen tempranamente en sus familias, constituyendo un aporte a la economía familiar al solventar gastos que corresponderían a los padres. Por otro lado, a pesar de no haberse observado prácticas de ahorro de manera significativa en este estrato es posible que estas existan pero no como conductas de ahorro formales. Denegri et al. (2008) indican la existencia en los niveles bajos de una tendencia al ahorro por vías no formales, ya sea ahorrando el dinero en la casa o dejándolo en poder de los padres, constituyéndose así en un mecanismo de ahorro precario pero inmediato para afrontar situaciones de carencia. Estas formas de ahorro y uso del dinero de los niños de NSE bajo se enmarcan dentro de una economía centrada en la familia y orientada a sobrellevar las carencias cotidianas, la cual contrasta con la tendencia a la acumulación del dinero y al uso del consumo desde una perspectiva más hedónica e individualista que es observada en niveles más altos (Denegri, et al., 2008).

Los niños provenientes de los niveles socioeconómicos medios reciben dinero todos los días en mayor porcentaje que los otros estratos, pero a la vez son quienes registran un mayor porcentaje de recepción mensual. Ello podría indicar la coexistencia de dos mecanismos de socialización económica antagónicos entre sí. Por una parte, la entrega diaria que no estimula los hábitos de ahorro y planificación, y por otra parte, la entrega mensual que podría ser equivalente al concepto de mesada, estrategia que desde la literatura emerge como una de las más eficientes para la socialización económica (Denegri, Palavecinos y Gempp, 2004; Denegri et al., 2008). Este resultado refleja probablemente los conflictos actuales al interior de las familias de clase media en torno a la forma de educar económicamente a sus hijos. Por una parte, tenemos a padres que esperan transmitir a sus hijos los valores de mensura, organización, austeridad y sacrificio que han caracterizado tradicionalmente a las clases medias latinoamericanas, y por otra parte, se ven impulsados por una sociedad de consumo que les indica que se es mejor padre o madre cuando se satisfacen todas las necesidades de los niños, dentro de las cuales el consumo parece ser una de las más relevantes de acuerdos de las sociedades postmodernas (Bauman, 2007). En este segmento, se observa una mayor autonomía 
en los niños para decidir qué hacer con su dinero en forme independiente de los padres, por lo que la influencia de ellos no es tan significativa. El dinero recibido suele destinarse a vestimenta y regalos. En cuanto a la tendencia al ahorro, esta es mayor en el NSE medio-alto, no así entre los niños de NSE medio-bajo, lo cual según Denegri et al. (2008), podría reflejar una mayor tendencia hacia el consumo impulsivo en los niños de NSE medio-bajo al momento de comprar lo que podría tener impacto en una mayor dificultad para desarrollar prácticas de ahorro sistemáticas. En general, si bien ambos grupos consideran que el dinero es algo importante, esta apreciación es reportada con más frecuencia entre los niños de NSE medio-bajo, lo que indica una postura de mayor tendencia hacia el consumismo en este segmento y es coherente con estudios previos que señalan que sería el estrato medio-bajo el que se encuentra en mayor vulnerabilidad frente a las presiones de la sociedad de consumo y por ende en mayor riesgo de comportamientos financieros poco reflexivos (Denegri, Palavecinos, Gempp y Caprile, 2005).

Los niños de NSE alto, al igual que en los otros estratos reciben dinero cuando lo piden y en segundo lugar de manera mensual, y si bien manifiestan autonomía en la decisión de su uso, son quienes también más deciden discutiéndolo con sus padres, lo que indica un estilo de socialización económica en las familias más reflexivo y orientador. El dinero recibido es destinado al consumo de libros, revistas y golosinas. Estos resultados complementan lo encontrado por estudios anteriores (Denegri, et al., 2008), los cuales indican que los tweens de niveles altos gastan su dinero en actividades recreativas y en bienes durables, a diferencia de los niños de otros estratos, explicado principalmente por el dinero que reciben de forma periódica, como la mesada, y el dinero auto gestionado, mediante prácticas de generación de dinero, como venta de ropa, CDs o productos hechos por ellos. Se observan las prácticas de ahorro descritas en otras investigaciones, las cuales son características de este estrato, dada la accesibilidad a sistemas formales de ahorro, propiciados por los padres, quienes les abren cuentas en instituciones bancarias desde pequeños. La opinión que estos niños tienen sobre el dinero es más bien pragmática que valorativa, siendo el segmento que en mayor porcentaje señala que este no es importante. Es probable que bajo esta opción subyazca una característica encontrada en otros estudios sobre la valoración dada al dinero en los segmentos altos, dicha valoración no está dada por su posesión en sí sino como un vehículo de acceso a otros satisfactores por lo que es muy importante su autogestión, administración y ahorro (Kiyosaki y Lechter, 2007).

Denegri (2010), al hacer una revisión de diversos modelos, explica que el entorno cultural, las experiencias económicas y el nivel socioeconómico, son factores que influyen significativamente en la aproximación de los niños al mundo económico. Esto explicaría por qué se observaron diferencias entre los distintos niveles socioeconómicos, de manera consistente con otros estudios.

Otro factor asociado son la reproducción y transmisión de las pautas de socialización económica que caracterizan a cada nivel, y la disponibilidad de dinero del que sus integrantes disponen. Por ejemplo, Denegri et al. (2008) indican que los niños 
de NSE alto reproducen las conductas de generación y acumulación de capital aprendidas en sus familias, mientras que los niños de NSE bajo continúan patrones de dependencia y subordinación laboral presentes en sus familias, explicando las presencia del ahorro en los niveles más altos.

El no haber encontrado una relación entre el género y las variables estudiadas, no quiere decir necesariamente que ambos grupos sean homogéneos en sus hábitos de consumo, sino que, en esta investigación, no fueron abordados aquellos aspectos que diferencian a los hombres de las mujeres en sus hábitos de consumo. Respecto a este aspecto, se sugiere, para futuros estudios indagar si existe una relación entre el género y las actitudes atribuidas al dinero, ya que de acuerdo a estudios realizados en estudiantes universitarios (Gasiorowska, 2009; Santa María y Gómez, 2005) es muy probable encontrar resultados significativos en esa dimensión. Por otro lado, un estudio longitudinal podría ser útil para observar la evolución de los hábitos descritos en esta investigación.

\section{CONCLUSIONES}

A partir de los resultados encontrados, es posible concluir que todos los niños y niñas reciben dinero de forma regular y cada vez que lo piden. Por su parte, la práctica de ahorro es transversal a los niveles socioeconómicos medio alto y alto. Se observan perfiles diferenciados entre los tweens de cada nivel socioeconómico, de acuerdo a la forma en que emplean el dinero y sus prácticas de ahorro. Los niños de NSE bajo reciben dinero de sus padres de manera semanal, siendo ellos quienes influyen en la decisión de cómo usarlo, y lo que reciben es destinado principalmente a gastos familiares como la compra de cosas necesarias para la escuela y la casa. Los niños de NSE medio reciben dinero de forma mensual, cuyo uso es decidido independientemente de los padres, destinándolo a vestimenta y regalos. Los niños de NSE alto discuten con sus padres en qué usarán el dinero que ellos les dan de manera mensual y destinan el uso al consumo de libros, revistas y golosinas.

\section{REFERENCIAS}

Bauman, Z. (2007). Vida de consumo. Buenos Aires: Fondo de Cultura Económica.

CNTV, \& McCann-Ericsson. (2003). Informe 8/13: Los Tweens Chilenos. Santiago: Maval.

Denegri, M. (2010). Introducción a La Psicología Económica. Retrieved from www. eumed.net/libros/2010b/681/

Denegri, M. \& Martínez, G. (2004). ¿Ciudadanos o consumidores? Aportes constructivistas a la educación para el consumo. PAIDEIA. Revista de Educación, 37, 101-116.

Denegri, M., Palavecinos, M. \& Gempp, R. (2004). Socialización económica: un estudio descriptivo de las estrategias y prácticas socializadoras y de alfabetización económica en familias de una ciudad multifinanciera. Informe de Avance. Proyecto Fondecyt $\mathrm{N}^{\mathrm{o}} 1030271$. 
Denegri M., Palavecinos M., Gempp R. \& Caprile C. (2005). Socialização econômica em famílias chilenas de classe média: educando cidadãos ou consumidores? Psicología e Sociedade, 17(2), 88 -98.

Denegri, M., Lara, M. Á., Córdova, G., \& Del Valle, C. (2008). Prácticas de ahorro y uso del dinero en pre adolescentes (Tweens) chilenos. Revista Universum, Universidad de Talca, 1(23), 24-38.

Furnham, A., \& Argyle, M. (1998). The Psychology of Money. London: Routlegde.

Gasiorowska, A. (2009). The Relationship of Income and Money Attitudes to Subjective Assessment of Financial Situation. Paper presented at the Selected Proceedings of the IAREP/SABE 2008, LUISS in Rome.

Guber, S., \& Berry, J. (1993). Marketing to and through Kids. New York.: McGraw - Hill.

Hernández, R., Fernández, C., \& Baptista, P. (1994). Metodología de la Investigación. Colombia: McGraw Hill.

Kiyosaki, R. y Lechter, S. ( 2007). Lo mejor de Padre Rico. Secretos para el éxito. Buenos Aires: V\&R.Ediciones.

McNeal, J. (2000). Children as Consumers of Commercial and Social Products. Washington: Organización Panamericana de la Salud.

McNeal, J. \& Yeh, C. (1990). Taiwanese children as consumers. Asia - Pacific Internacional Journal of Marketing, 2, 32 - 43.

McNeil, J. \& Yeh, C. (1997). Development of consumer behavior patterns among Chinese children. Journal of Consumer Marketing, 14, 45-49.

McNeil, J. (1965). Dimensions of Consumer Behavior. New York: Meredith Publishing Company.

Moschis, G. P. (1987). Consumer Socialization, a Life Cycle Perspective. Lexington. : Lexington Books, DC HealthCompany.

Ozgen, O. (2003). An Analysis of Child Consumers in Turkey. International Journal of Consumer Studies, 27(366-380).

Prensky, M. (2001). Digital Natives, Digital Immigrants. On the Horizon, University Press, 9(5), 1-6.

Rose, G. (1999). Consumer Socialization, Parental Style, and Developmental Timetables in the United States and Japan. Journal of Marketing, Vol 63, 105-119.

Santa María, C., \& Gómez, J. (2005). Actitudes hacia el dinero en jóvenes de 18 a 23 años. Revista Electrónica de Psicología “Ajayu”, Universidad Católica Boliviana "San Pablo" 3(1). 


\section{Tablas}

Tabla 1. Relación entre NSE y frecuencia de entrega de dinero

\begin{tabular}{|c|c|c|c|c|c|c|}
\hline \multirow{2}{*}{ Frecuencia } & \multicolumn{4}{|c|}{ Nivel socioeconómico } & \multirow[b]{2}{*}{$X^{2}$} & \multirow[b]{2}{*}{$p$} \\
\hline & Bajo & Medio-bajo & Medio-alto & Alto & & \\
\hline Todos los días & $10,1 \%$ & $11,5 \%$ & $11,8 \%$ & $1,2 \%$ & 8,331 & $0,040^{*}$ \\
\hline Semanalmente & $22 \%$ & $13,5 \%$ & $6,3 \%$ & $11,5 \%$ & 12,310 & $0,006^{* *}$ \\
\hline Mensualmente & $12,8 \%$ & $31,1 \%$ & $18,9 \%$ & $22,6 \%$ & 13,165 & $0,004^{* *}$ \\
\hline Sólo cuando lo pido & $52,3 \%$ & $41,2 \%$ & $53,5 \%$ & $48,8 \%$ & 5,068 & 0,167 \\
\hline
\end{tabular}

Nota. $*=\mathrm{p}<.05 ; * *=\mathrm{p}<.01$

Tabla 2. Relación entre género y frecuencia de entrega de dinero

\begin{tabular}{lcccc}
\hline \multirow{2}{*}{ Frecuencia } & \multicolumn{4}{c}{ Género } \\
\cline { 2 - 3 } & Hombres & Mujeres & $X^{2}$ & $\mathrm{p}$ \\
\hline Todos los días & $11,8 \%$ & $7,1 \%$ & 3,109 & 0,078 \\
Semanalmente & $16,7 \%$ & $10,8 \%$ & 3,370 & 0,066 \\
Mensualmente & $27,2 \%$ & $17,1 \%$ & 6,962 & $0,008^{* *}$ \\
Sólo cuando lo pido & $38,2 \%$ & $58,3 \%$ & 19,054 & $0,000^{* *}$ \\
\hline
\end{tabular}

Nota. $*=\mathrm{p}<.05 ; * *=\mathrm{p}<.01$

Tabla 3. Relación entre NSE y uso del dinero

\begin{tabular}{lcccccc}
\hline \multirow{2}{*}{ Uso del dinero } & \multicolumn{5}{c}{ Nivel socioeconómico } & \\
\cline { 2 - 5 } & Bajo & Medio-bajo & Medio-alto & Alto & X & p \\
\hline Ahorro & $38,5 \%$ & $54,7 \%$ & $66,9 \%$ & $65,5 \%$ & 22,828 & $0,000^{* *}$ \\
Comprar regalos & $3,7 \%$ & $0,7 \%$ & $2,4 \%$ & $2,4 \%$ & 2,791 & 0,425 \\
Comprar cosas para el colegio & $23,9 \%$ & $8,1 \%$ & $1,6 \%$ & $2,4 \%$ & 42,658 & $0,000^{* *}$ \\
Comprar cosas para la casa & $6,4 \%$ & $2 \%$ & $0,8 \%$ & $2 \%$ & 11,314 & $0,010^{* *}$ \\
Comprar otras cosas & $27,5 \%$ & $35,8 \%$ & $29,9 \%$ & $33,3 \%$ & 2,313 & 0,510 \\
\hline
\end{tabular}

Nota. $*=p<.05$

Tabla 4. Relación entre NSE y uso del dinero en otras cosas

\begin{tabular}{|c|c|c|c|c|c|c|}
\hline \multirow{2}{*}{ Uso del dinero } & \multicolumn{4}{|c|}{ Nivel socioeconómico } & \multirow[b]{2}{*}{$X^{2}$} & \multirow[b]{2}{*}{$p$} \\
\hline & Bajo & Medio-bajo & Medio-alto & Alto & & \\
\hline Juegos y Juguetes & $14,7 \%$ & $20,3 \%$ & $8,7 \%$ & $11,9 \%$ & 27,229 & $0,241^{*}$ \\
\hline Vestimenta & $2,8 \%$ & $4,7 \%$ & $7,1 \%$ & $4,8 \%$ & & \\
\hline Regalos & $0,9 \%$ & $4,1 \%$ & $0,0 \%$ & $0 \%$ & & \\
\hline Golosinas & $8,3 \%$ & $5,4 \%$ & $12,6 \%$ & $13,1 \%$ & & \\
\hline Libros y revistas & $0,9 \%$ & $1,4 \%$ & $1,6 \%$ & $3,6 \%$ & & \\
\hline
\end{tabular}

Nota. $*=p<.05$ 
Tabla 5. Relación entre género y uso del dinero

\begin{tabular}{lcccc}
\hline \multirow{2}{*}{ Uso del dinero } & \multicolumn{4}{c}{ Género } \\
\cline { 2 - 3 } & Hombres & Mujeres & $X^{2}$ & $\mathrm{p}$ \\
\hline Ahorro & $60,5 \%$ & $52,1 \%$ & 3,385 & 0,066 \\
Comprar regalos & $1,8 \%$ & $2,5 \%$ & 0,311 & 0,577 \\
Comprar cosas para el colegio & $7,9 \%$ & $10,0 \%$ & 0,634 & 0,426 \\
Comprar cosas para la casa & $1,8 \%$ & $2,9 \%$ & 0,688 & 0,407 \\
Comprar otras cosas & $29,8 \%$ & $33,8 \%$ & 0,042 & 0,362 \\
\hline
\end{tabular}

Tabla 6. Relación entre género y uso del dinero en otras cosas

\begin{tabular}{|c|c|c|c|c|}
\hline \multirow{2}{*}{ Uso del dinero } & \multicolumn{2}{|c|}{ Género } & \multirow[b]{2}{*}{$\mathrm{X}^{2}$} & \multirow[b]{2}{*}{ C } \\
\hline & Hombres & Mujeres & & \\
\hline Juegos y Juguetes & $18,9 \%$ & $10,0 \%$ & $26,732^{\star *}$ & 0,232 \\
\hline Vestimenta & $0,9 \%$ & $8,8 \%$ & & \\
\hline Regalos & $0,4 \%$ & $2,5 \%$ & & \\
\hline Golosinas & $8,8 \%$ & $10,0 \%$ & & \\
\hline Libros y revistas & $0,9 \%$ & $2,5 \%$ & & \\
\hline
\end{tabular}

Nota. $* *=\mathrm{p}<.01$.

Tabla 7. Relación entre NSE y decisión de uso

\begin{tabular}{|c|c|c|c|c|c|c|}
\hline Decide Solo & $67,6 \%$ & $88,5 \%$ & $80 \%$ & $78,6 \%$ & $32,177^{* *}$ & 0,254 \\
\hline Conversando con sus padres & $16,7 \%$ & $8,1 \%$ & $15,2 \%$ & $16,7 \%$ & & \\
\hline Padres dicen en qué gastar & $11,1 \%$ & $2,7 \%$ & $0,8 \%$ & $1,2 \%$ & & \\
\hline No recibe dinero & $4,6 \%$ & $0,7 \%$ & $4 \%$ & $3,6 \%$ & & \\
\hline
\end{tabular}

Nota. $* *=\mathrm{p}<.01$.

Tabla 8. Relación entre NSE e ideas sobre el dinero

\begin{tabular}{|c|c|c|c|c|c|c|}
\hline \multirow{2}{*}{ Idea sobre del dinero } & \multicolumn{4}{|c|}{ Nivel socioeconómico } & \multirow[b]{2}{*}{$X^{2}$} & \multirow[b]{2}{*}{$p$} \\
\hline & Bajo & Medio-bajo & Medio-alto & Alto & & \\
\hline Sirve para comprar & $64,2 \%$ & $37,8 \%$ & $46,5 \%$ & $59,5 \%$ & 21,251 & $0,000^{* *}$ \\
\hline Es importante & $21,1 \%$ & $43,2 \%$ & $33,1 \%$ & $15,5 \%$ & 25,290 & $0,000^{* *}$ \\
\hline Es bueno tener & $1,8 \%$ & $3,4 \%$ & $1,6 \%$ & $2,4 \%$ & 1,141 & 0,767 \\
\hline Es algo que hace feliz & $6,4 \%$ & $4,1 \%$ & $3,1 \%$ & $0 \%$ & 5,749 & 0,124 \\
\hline No es importante & $9,2 \%$ & $10,1 \%$ & $14,2 \%$ & $21,4 \%$ & 7,897 & $0,048^{*}$ \\
\hline
\end{tabular}

Nota. $*=\mathrm{p}<.05 ; * *=\mathrm{p}<.01$ 
CONCEPCIONES SOBRE DINERO Y DECISIONES SOBRE SU USO EN TWEENS

\section{NOTA ACERCA DE LOS AUTORES}

Esta investigación fue financiada por el Proyecto DI-112007, adjudicado por el Centro de Excelencia en Psicología Económica y del Consumo, Universidad de la Frontera.

\section{Dra. Marianela Del Carmen Denegri Coria}

Profesora Titular, Departamento de Psicología, Universidad de La Frontera, Temuco, Chile. Titulada de Psicología en la Pontificia Universidad Católica de Chile, en el año 1995 recibe el grado de Doctora en Psicología por la Universidad Autónoma de Madrid. Actualmente dirige el Centro de Investigación en Psicología Económica y del Consumo (CEPEC). Datos de contacto: Av. Francisco Salazar 01145, Temuco, Chile, correo mdenegri@ufro.cl, fono: 56-45-325614.

\section{Lic. Javier Álvarez Gutiérrez}

Licenciado en Psicología, Universidad de La Frontera, actualmente cursa el Mg. en Psicología, Universidad de La Frontera. Sus datos de contacto son los siguientes: Av. Francisco Salazar 01145, Temuco, Chile, correo j.alvarez02@ufromail.cl.

\section{Mg. Jocelyne Sepúlveda Aravena}

Titulada de Psicología, Universidad de La Frontera, en el año 2010 recibe el grado de Magíster en Psicología por la Universidad de La Frontera. Investigadora del Centro de Investigación en Psicología Económica y del Consumo (CEPEC). Actualmente se desempeña como Encargada del Área de Competencias Genéricas del Centro de Innovación Profesional, Universidad de La Frontera. Datos de contacto: Av. Francisco Salazar 01145, Temuco, Chile, correo jksepulveda@ ufro.cl, fono: 56-45-734184. 